\title{
The effect of the environment conditions on the prediction of flammable cloud dispersion.
}

\author{
A. M. Schleder \& M. R. Martins \\ Naval Architecture and Ocean Engineering Department, Risk Assessment Laboratory, University of São \\ Paulo, São Paulo, Brazil
}

E. Pastor \& E. Planas

Department of Chemical Engineering, Centre for Technological Risk Studies, Universitat Politècnica de Catalunya - BarcelonaTech, Barcelona, Spain

\section{ABSTRACT}

In order to quantify the damage caused by undesired events involving leakages of flammable materials, specific models are used to analyze the spills or jets of gas and liquid, gas dispersion, explosions and fires. The main step of this analysis is to estimate the concentration, in space and time, of the vapor cloud of hazardous substances released into the atmosphere; the purpose is to determine the area where a fire or explosion might occur and the quantity of flammable material in that area. Recently with the computational advances, CFD tools are used to short and medium range gas dispersion scenarios, especially in scenarios where there is a complex geometry. However, the accuracy of the simulating strongly depends on the boundary conditions. Therefore, this study investigates the sensitivity degree of the prediction of cloud dispersion to changes in values of wind speed, ambient temperature, atmospheric pressure and ground roughness. This paper con-tributes to an appropriate assessment of the effects of these environment conditions to perform an accurate dispersion simulation using CFD tools and therefore contributes to a more effective analysis of the consequences.

\section{REFERENCES}

Anderson, J. D. Jr. 1995. Computational Fluid Dynamics - The basics with applications. Maryland: McGraw-Hill. pp. 547.

CCPS - Center for Chemical Process Safety, 1995. Understanding Atmospheric Dispersion of Accidental Releases. New York: Center for Chemical Process Safety.

Coldrick, S., Lea, C. J., \& Ivings, M. J., 2009. Validation database for evaluating vapor dispersion model for safety analysis of LNG facilities - Review. The fire protection research foundation. Health and Safety Laboratory - HSE.

Cormier, B. R. 2008. Application of computational fluid dynamics for LNG vapor dispersion. Journal of Loss Prevention in the Process Industries. pp. 332-352.
Dharmavaram, S., Hanna, S. R., \& Hansen, O. R. 2005. Consequence Analysis-Using a CFD model for industrial Sites. Process Safety Progress, 24, pp. 316-327.

Gant, S. E., Kelsey, A., McNally, K., Witlox, H. 2013. Sensitivity Analysis of Dispersion Models for Jet Releases of Dense-Phase Carbon Dioxide. Chemical Engineering Transactions, 31, pp. 1-6.

Gavelli, F., Scott, D. G., \& Hansen, O. R. 2011. Evaluating the potencial for overpressures from the ignition of a LNG vapor cloud during offloading. Journal of Loss Prevention in the process industries, 24, pp. 908-915.

GexCon AS. 2014. FLACS Software - version 10.1.

HSE - Health and Safety Executive. 2010. Review of FLACS version 9.0 - Dispersion modelling capabilities. Available at: http://www.hse.gov.uk/research/rrpdf/rr779.pdf, accessed on January, 2013.

Launder, B. E., \& Spalding, D. B. 1974. The numerical computation of turbulent flows., 3, pp. 269-289.

Middha, P. and Hansen, O. R. 2009. Using computational fluid dynamics as a tool for hydrogen safety studies. Journal of Loss Prevention in the Process Industries. Vol. 22, pp. 295302.

Pandya, N., Gabas, N., Marsden, E. 2012. Sensitivity analysis of Phast's atmospheric dispersion model for three toxic materials (nitric oxide, ammonia, chlorine). Journal of Loss Prevention in the Process Industries(25), pp. 20-32.

Plasmans, J., Donnat, L., Carvalho, E., Debelle, T., Marechal, B., Baillou, F. 2012. Challenges with the use of CFD for major accident dispersion modeling. Process Safety Progress. Vol.00, pp. 1-5. doi: 10.1002/prs.11571.

Schleder, A. M., Martins, M. R. 2013. The use of Integrals and CFD tools to evaluate the cloud dispersion of flammable and toxic substances leakages. In: European Safety and Reliability Congress - ESREL, 2013, Amsterdã. Proceedings of ESREL 2013 - European Safety and Reliability Congress. pp. 1-8.

Schleder, A. M., Martins, M. R., Pastor, E., Planas, E. 2014. The effect of the computational grid size on the prediction of a flammable cloud dispersion. In: International Conference on Ocean, Offshore and Arctic Engineering, 2013, San Francisco, California, USA. Proceedings of the ASME 2014 33th International Conference on Ocean, Offshore and Arctic Engineering, 2014. pp. 1-8.

TNO - The Netherlands Organization of Applied Scientific Research 2005. Methods for the calculation of physical effects due to releases of hazardous materials (liquids and gases) Yellow Book (Third Edition ed.). Committee for the prevention of disasters. 


\title{
The effect of the environment conditions on the prediction of flammable cloud dispersion.
}

\author{
A. M. Schleder \& M. R. Martins \\ Naval Architecture and Ocean Engineering Department, Risk Assessment Laboratory, University of São \\ Paulo, São Paulo, Brazil \\ E. Pastor \& E. Planas \\ Department of Chemical Engineering, Centre for Technological Risk Studies, Universitat Politècnica de \\ Catalunya - BarcelonaTech, Barcelona, Spain
}

\begin{abstract}
In order to quantify the damage caused by undesired events involving leakages of flammable materials, specific models are used to analyze the spills or jets of gas and liquid, gas dispersion, explosions and fires. The main step of this analysis is to estimate the concentration, in space and time, of the vapor cloud of hazardous substances released into the atmosphere; the purpose is to determine the area where a fire or explosion might occur and the quantity of flammable material in that area. Recently with the computational advances, CFD tools are used to short and medium range gas dispersion scenarios, especially in scenarios where there is a complex geometry. However, the accuracy of the simulating strongly depends on the boundary conditions. Therefore, this study investigates the sensitivity degree of the prediction of cloud dispersion to changes in values of wind speed, ambient temperature, atmospheric pressure and ground roughness. This paper contributes to an appropriate assessment of the effects of these environment conditions to perform an accurate dispersion simulation using CFD tools and therefore contributes to a more effective analysis of the consequences.
\end{abstract}

\section{INTRODUCTION}

Specific models are used to evaluate the release and dispersion of flammable substances when an undesired event occurs; the determination of the dispersion features is essential to model the consequences such as fires and explosions. The consequence analysis is used to define the extent and nature of effects caused by such events and thus is of great help when quantifying the damage caused.

Dispersion models estimate the evolution and the features of the cloud, such as concentration, temperature, velocity and dimensions as a function of time and position. In the case of flammable substances, these models facilitate the prediction of an area where a fire or explosion might occur and the quantity of flammable material in that area.

Nowadays, the use of numerical methods associated with different algorithms of computational fluid dynamics (CFD) to determine the concentration of the vapor cloud of hazardous substances released into the atmosphere, in space and time, has grown considerably (Comier 2008, Middha 2009, Dharmavaram, 2005). CFD is found in some commercial software tools such as CFX, FLACS and FLUENT. The CFD tools transform the governing equations of the fundamental physical principles of fluid flow in discretized algebraic forms, which are solved to find the flow field values in time and space (Anderson, 1995).

CFD tools have proven promising to perform analyzes of consequences in environments with complex geometry, as in the comparative study about the use of integral and CFD tools to evaluate cloud dispersion reported by Schleder \& Martins (2013). Nevertheless, as reported by Plasmans et al. (2012), previous studies have shown that consequences analysis using CFD are frequently not easily reproduced and, in many occasions, large differences can arise between the simulation results when working with different tools and/or different CFD analysts to assess the same scenario. These problems stem from the fact that the simulation results can be very sensitive to the wide range of computational parameters that must be set by the user; for a typical simulation, the user needs to select the variables of interest, turbulence models, computational domain, computational mesh, methods of discretization, convergence criteria and boundary conditions among others. The boundary conditions, especially the atmospheric conditions, affect directly the dispersion; however, in most cases, the conditions are not known and approximate values are used to perform the consequences analyses.

In this context, the study presented here intends to investigate the effects of variations on boundary conditions on the simulation of a cloud dispersion. 
This study is part of a research project that aims to provide key information for decision making about the use of CFD tools on cloud dispersion evaluation for different scenarios of interest, such as those containing barriers to dispersion; and therefore contributing to optimize the accuracy of the results.

In order to perform this analysis, the same scenarios used in a previous study of grid dependence are used (Schleder et al., 2014) in this case to assess the influence of variations on boundary conditions on the predicted values of the cloud features. The variables of interest have been ambient temperature, atmospheric pressure, wind speed and ground roughness. Two scenarios are presented: a former scenario of a jet release of dense gas in open and flat terrain and a similar release with the presence of a fence.

\subsection{Computational Fluid Dynamics (CFD)}

The physical aspects of any fluid flow are governed by three principles: mass is conserved, Newton's second law is fulfilled (also referred as momentum equation) and energy is conserved; these principles are expressed in integral equations or partial differential equations (continuity, momentum and energy equations), being the most common form the Navier-Stokes equations for viscous flows and the Euler equations for inviscid flows.

The CFD tools transform these governing equations of the fundamental physical principles of fluid flow in discretized algebraic forms, which are solved to find the flow field values in time and/or space. Thus, the results obtained by CFD are a set of data which represent the flow field characteristics at discrete points in time and/or space. The application of CFD tools are related with the computational advances, which explains the growing use of CFD in the recent years (Anderson, 1995).

The boundary and initial conditions of the flow dictate the particular solution obtained from the governing equations, and as such the solution of the governing equations must provide a suitable representation of the boundary conditions.

The software FLACS was used to perform this study (GexCon AS, 2013); FLACS incorporates a water-based model for the simulation of pool spreading and vaporization, and the Reynolds Averaged Navier-Stokes (RANS) equations for the simulation of vapor cloud dispersion.

The pool vaporization is evaluated based on heat transfer from the substrate, solar radiation, convective heat transfer from the air, local wind speed, turbulence levels and local vapor pressure. All these variables are calculated at each time step and locally, for each grid cell (Gavelli et al. 2011). The cloud concentration also will be influenced by atmospheric turbulence, atmospheric stability and density changes. FLACS models flow in the atmospheric boundary layer by profiles of wind, temperature and turbu- lence parameters on the inlet boundaries (GexCon AS, 2013).

As presented by Gavelli et al. (2011), the model available in FLACS solves Reynolds Averaged Navier-Stokes (RANS) equations based on the standard k- $\varepsilon$ model of Launder \& Spalding (1974). According to HSE (2010), RANS approach is widely accepted and documented; it is based on the concept of separating the fluid velocity components and scalar quantities (pressure, temperature, concentration) into mean and fluctuating components, then transport equations are used to evaluate the model. The standard k- $\varepsilon$ model of Launder \& Spalding (1974) presents a turbulence model in which the magnitudes of the two turbulence quantities are evaluated: the turbulence kinetic energy $\mathrm{k}$ and its dissipation rate $\varepsilon$, which are calculated from transport equations solved simultaneously with those governing the mean flow behavior (Launder \& Spalding 1974).

\subsection{The effects of the atmospheric conditions}

As reported by CCPS (1995), to analyze an accidental release it is necessary to define the governing conditions of the discharge scenario and environment. The items that can define these conditions are source term characteristics, environmental conditions, types of releases, possible source scenarios and possible dispersion mechanisms. The release is usually described separating the region analyzed in three sections: first the discharge section, where the release is almost independent of the environmental conditions and as such the features of the source term define this region; next the intermediary section where both source and environmental conditions are significant in the modeling and the last section, where environmental conditions dominate the process of dispersion. In this paper the focus is on the effects of environmental conditions in the whole dispersion process.

If the substance released presents density higher than air, the first stage of the dispersion will occur as dense gas and when the cloud dilutes enough to its density be equal to the air's, the dispersion will occur as passive or neutral dispersion. In the dense gas dispersion phase, the cloud will be making descent movements until it reaches the ground and then will spread radially under influence of the gravitational forces, hence having the dense cloud the horizontal dimension greater that the vertical dimension. The vertical dimension will be higher in the extremities of the cloud due to the air resistance (TNO, 2005).

After this stage, when the cloud has density equal to the air's, the passive dispersion occurs which is governed by the environmental conditions, mainly by wind and atmospheric stability (TNO, 2005).

The atmospheric instability occurs because of the wind flow and the air displacement between differ- 
ent layers due to the temperature difference between these layers. This turbulence generates eddies with different sizes; eddies smaller than the cloud disperse the cloud and increase the cloud size (there is no effect on position of the cloud), eddies much bigger than the cloud merely move the cloud (there is no effect on form neither on size) and eddies with the same size of the cloud change the cloud form and increase its contour (TNO, 2005).

\section{BASELINE SCENARIO}

As previously mentioned, the baseline scenario in this study is the same used in a previous study of grid dependence (Schleder et al. 2014) where two trials of the field tests performed by Health and Safety Laboratory (HSL) at the HSL laboratories in Buxton, England (Butler \& Royle, 2001) were chosen to perform the sensitivity analysis.

The HSL trials consisted of liquefied propane continuous releases at rates up to $4.9 \mathrm{~kg} / \mathrm{s}$, at a height of $1.5 \mathrm{~m}$; during the release a biphasic jet was formed, however there was no pool formation, since the liquid present in the jet evaporated before reaching the ground. The concentration of propane in the resulting vapor cloud was monitored by gas sensors to determine the cloud features at different distances from the release point. The trials set-up involved a liquefied propane storage facility, a release system and a discharge area in which the vapor clouds were produced. The discharge length was aligned with the prevailing wind.

Sensors were placed over a $600 \mathrm{~m}^{2}$ area $(100 \mathrm{~m}$ in downwind direction and $6 \mathrm{~m}$ in crosswind direction), located within the gas dispersion site; they were located at heights of $0.20,0.85$ or $1.50 \mathrm{~m}$ above the ground on the first $40 \mathrm{~m}$ of the centerline of the site and at a height of $0.20 \mathrm{~m}$ in arcs around the release point.

In order to investigate the influence of an obstruction, in some of the trials a fence was placed in the cross wind direction in the path of the vapor flow. A $1 \mathrm{~m}$ high fence was chosen to be a suitable obstruction; using this height, the top of the fence was approximately in the middle of the gas cloud height, allowing a significant volume of gas to flow unobstructed, whilst at the same time providing an obstruction for the lower part of the cloud. The fence was $20 \mathrm{~m}$ long, positioned $15 \mathrm{~m}$ from the release nozzle, perpendicular to the centerline of the trials site. It was centered so that there was $10 \mathrm{~m}$ of fence at either side of the centerline. More details about the field tests are reported in Butler \& Royle (2001) and Schleder et al. (2014).

In the previous study (Schleder et al., 2014) trials 8 and 11 of these field tests were selected as baseline scenarios (B1 and B2) to perform the CFD grid dependence analysis. The same trials were selected to perform the sensitivity analysis. The former test (B1 representing test 8 ) presents an unobstructed scenario while the other (B2 representing test 11) presents the scenario with a fence acting as an obstruction to cloud dispersion. The input parameters used to perform the simulations are presented in Table I.

Table I - Scenario conditions of baseline scenarios

\begin{tabular}{lccc}
\hline Variable & Unit & B1 & B2 \\
\hline Ambient temperature & oC & 14.5 & 17.5 \\
\hline Atmospheric pressure & bar & 1 & 1 \\
\hline Wind speed & $\mathrm{m} / \mathrm{s}$ & 3.0 & 5.0 \\
\hline Pasquill class & - & $\mathrm{D}$ & $\mathrm{D}$ \\
\hline Wind direction & o & $195-225$ & $110-225$ \\
\hline Relativity humidity & $\%$ & 63 & 63 \\
\hline Ground roughness & $\mathrm{m}$ & 0.03 & 0.03 \\
\hline Temperature release & oC & 11.96 & 11.26 \\
\hline Pressure release & bar & 7.87 & 7.58 \\
\hline Discharge rate & $\mathrm{kg} / \mathrm{s}$ & $2.5 \pm 0.3$ & $3.4 \pm 0.3$ \\
\hline Discharge direction & - & horizontal & horizontal \\
\hline Release duration & $\mathrm{s}$ & 131 & 141 \\
\hline Discharge height & $\mathrm{m}$ & 1.5 & 1.5 \\
\hline
\end{tabular}

The domain and the grid of the baseline scenarios were built according to previous study (Schleder et al., 2014): an orthogonal base X, Y and Z was used, being the $X$ direction horizontal and parallel to wind, the $\mathrm{Y}$ direction perpendicular to the wind and horizontal and the $\mathrm{Z}$ direction vertical, being the point $(0,0,1.5)$ coinciding with the release point.

The domain was divided in three areas: the first one around the release point (micro grid), formed by the specific and adjacent cells where the leak takes place; the second, the prevailing grid formed by the area where the dispersion is expected (macro grid); and the third, the stretched area in the far field where no relevant concentrations are expected. The transitions among these areas are made gradually in order to obtain stable simulations; the cells are increases gradually from one region to another of the grid such as the maximum ratio between one cell and the next one is two.

The computational domain extended $170 \mathrm{~m}$ in the $\mathrm{X}$ direction (from $20 \mathrm{~m}$ upwind to $150 \mathrm{~m}$ downwind from the release point), $30 \mathrm{~m}$ in the $\mathrm{Y}$ direction (symmetric crosswind plan from the release point) and $10 \mathrm{~m}$ in the $\mathrm{Z}$ direction; the cells were initially represented by $1 \mathrm{~m} \times 1 \mathrm{~m} \times 0.5 \mathrm{~m}$ cuboids (forming the macro grid).

Concerning the micro grid dimensioning, the guidelines (GexCon AS, 2013) specify that the cross area of the expanded jet must be solved in only one cell and that the area of this cell should be larger than the area of the expanded jet but not larger than twice. Therefore, the jet area expected after the expansion at ambient pressure was estimated and the 
dimensions of the face cell across the jet defined so that the area fell between these limits. Thus, in B1 scenario, the width and height of the micro grid cells (the release cell and adjacents) were fixed at $0.15 \mathrm{~m}$ (as a function of the jet area expected after the expansion at ambient pressure) and, in order to maintain the aspect ratio smaller than 5, the length of the cells was fixed at $0.5 \mathrm{~m}$. In B2 scenario, the width and height of the micro grid cells were fixed at 0.17 $\mathrm{m}$ and the length of the cells was fixed at $0.86 \mathrm{~m}$. Once the dimensions of the cells around the leak were defined, cells nearby were smoothly increased to the macro grid resolution: in $\mathrm{z}$ direction, the transition zone between the micro grid and the macro grid is from $2 \mathrm{~m}$ to $5 \mathrm{~m}$ high; in y direction, this region has $12 \mathrm{~m}$ in the symmetrical crosswind plan from the release point; and in $\mathrm{x}$ direction, the macro grid begins $1 \mathrm{~m}$ after the release in downwind direction and $1 \mathrm{~m}$ after the release in upwind direction..

Lastly, in both scenarios, the macro grid was stretched in $\mathrm{X}$ direction away from the leakage point, (he length of cell growing continuously at a rate of 1.15 to provide a smooth growth with increasing distance from the source. The cells were stretched after $100 \mathrm{~m}$ from the leakage point because after this distance significant concentrations of gas are not expected. More details about the grid are reported by Schleder et al. (2014).

Taking into account that the focus of this study is on the dispersion of a cloud the main variable of interest was defined as the concentration of this cloud.

The results of the baseline scenarios are presented in Figure 1 and Figure 2 for scenarios B1 and B2 respectively; the blue lines represent experimental data and the green lines represent the simulated concentrations.

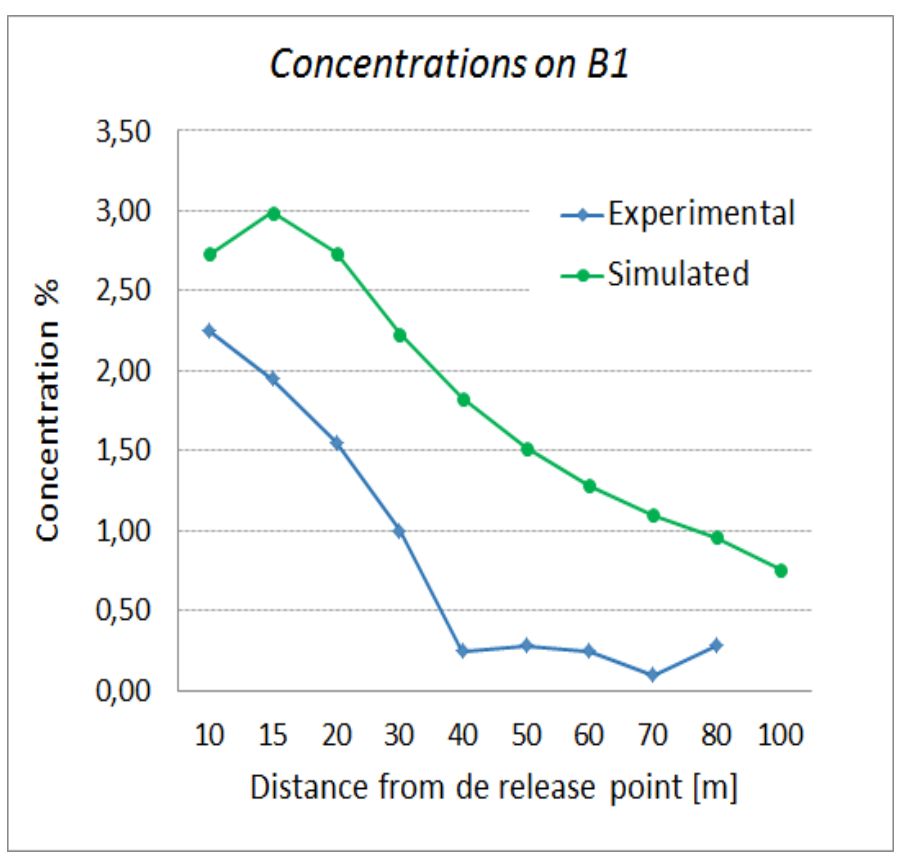

Figure 1 - Results of baseline scenario B1

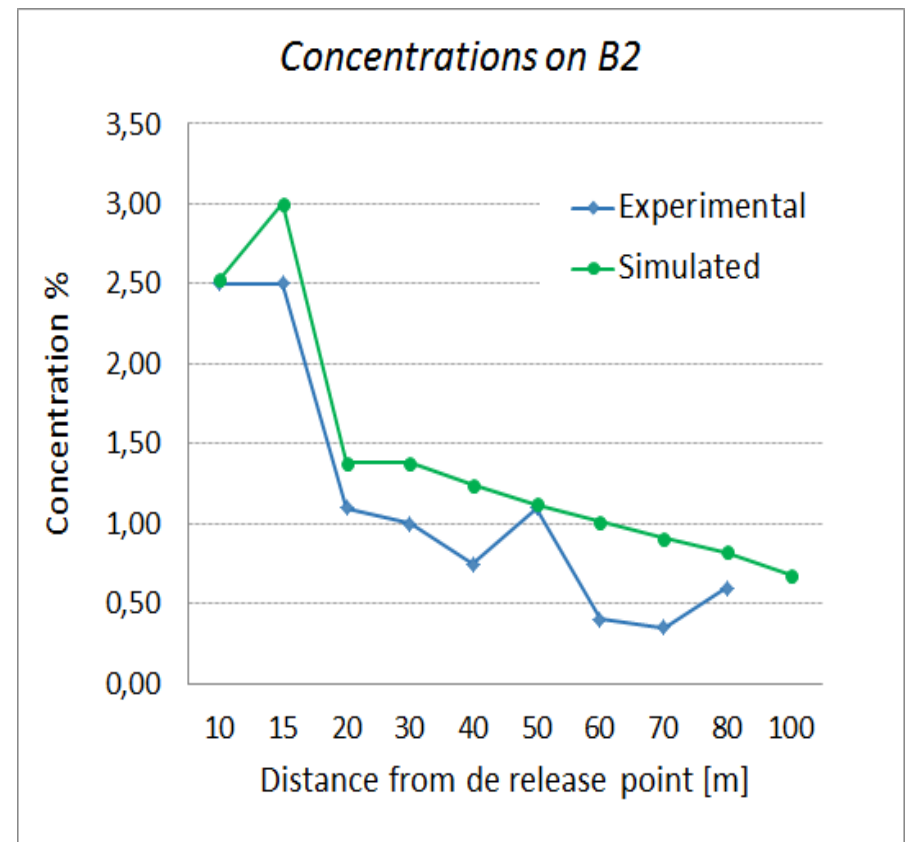

Figure 2 - Results of baseline scenario B2

The HSE in the Model Evaluation Protocol (MEP) recommends the use of a factor of 2 range to validation purposes of CFD models, the fraction of predictions should fall within a factor of two of the measurements (Coldrick et al., 2009); in the cases analyzed here, $60 \%$ of the simulated values fitted well to this range, the major discrepancies found are related to values of concentration smaller than $2 \%$ in scenario B1 that are over simulated by the model. The simulated concentrations concerning to baseline scenarios were reported and discussed in Schleder et al. (2014).

\section{SENSITIVITY ANALYSIS}

In CFD modeling, the analyst should choose a large number of parameters and these can affect significantly the results. As reported by Pandya, et al. (2012), there are three varieties of sensitivity analysis: local, global and screening methods. The local methods evaluate the effects in the outputs given each input varying one factor at a time around a baseline point; the global methods are more sophisticate and aim to evaluate quantitatively the influence of the entire range of inputs values on the outputs uncertainty. Finally, the screening methods that are based on computing for each input a number of incremental ratios, which are then averaged to assess the importance of the input (Pandya, et al., 2012).

The global and screening methods are comprehensive methods that assess the sensitivity of the models in more detail; however, these models deal with the variables as functions, therefore are more time consuming and require more complex tools for their development such as the software's used by Pandya, et al (2012) and Gant, et al. (2013). Considering that CFD models are computationally expensive and 
have a large range of possible inputs, the sensitivity analysis performed here will follow the local approach, which is less time consuming and can be executed with no need of specific software.

The objective of the sensitivity analysis is to verify which inputs affect more the results of the simulation, thus the first step is the choice of the variables subjected to sensitivity analysis. Next, these inputs are varied and the effects on the results regarding to concentration of the cloud are analyzed. Finally the degree of sensitivity is estimated.

As seen, the boundary conditions, especially the atmospheric conditions, affect directly the dispersion; however, in most cases the exact values of the parameters that characterized the conditions are not known and approximate values are used to perform the consequences analyses. In this sensitivity analysis the effects of variations in the wind speed, ambient temperature, atmospheric pressure and ground roughness on predicted concentrations are analyzed.

In order to perform the sensitivity analysis the influence of variations of up to $\pm 10 \%$ in the ambient temperature, wind speed, ground roughness and atmospheric pressure values was studied. Each variable was changed independently of the others: for example, when the ambient temperature was decreased by $10 \%$, the other variables remained the same as those defined in the baseline scenario (Table I).

The same approach was used for both baselines scenarios (B1 and B2); nine simulations were performed for each one: the former using the original values presented on Table I and one for each variation presented on Table II, in which the first column describes the variable of interest, the second is the variation applied over the original value of this variable and the last two the final value of each variable after increasing or decreasing by $10 \%$ for scenarios $\mathrm{B} 1$ and $\mathrm{B} 2$ respectively.

Table II - Variations in each scenario

\begin{tabular}{lcccc}
\hline Variable & Variation & Unit & B1 & B2 \\
\hline Ambient temperature & $-10 \%$ & ㅇ & 13.05 & 15.75 \\
\hline Ambient temperature & $+10 \%$ & ㅇ & 15.95 & 19.25 \\
\hline Wind speed & $-10 \%$ & $\mathrm{~m} / \mathrm{s}$ & 2.70 & 4.50 \\
\hline Wind speed & $+10 \%$ & $\mathrm{~m} / \mathrm{s}$ & 3.30 & 5.50 \\
\hline Ground roughness & $-10 \%$ & $\mathrm{~m}$ & 0,027 & 0,027 \\
\hline Ground roughness & $+10 \%$ & $\mathrm{~m}$ & 0,033 & 0,033 \\
\hline Atmospheric pressure & $-10 \%$ & bar & 0,90 & 0,90 \\
\hline Atmospheric pressure & $+10 \%$ & bar & 1,10 & 1,10 \\
\hline
\end{tabular}

The simulated values for concentration of each simulation were compared with the simulated values of the original baseline scenarios. Monitor points measuring concentration every $10 \mathrm{~m}$ following $\mathrm{X}$ direction were inserted in the simulations at three heights: $0.2,0.8$ and $1.5 \mathrm{~m}$ and thus the comparative analysis between the simulated concentrations of the original baseline scenarios and the simulations after the variation of each parameter was made for each distance and height.

Only the results obtained for the monitor points at 0.2 high are presented here due to space constraint, on Table III and Table IV. In these tables, the first column reports the distances from the monitor point to the release point, and the other columns report the maximum percentage variation between the simulated concentrations using the original values of the baseline scenario and the concentration simulated after the variation on each particular parameter. The symmetry of the results is analyzed posteriorly. For example, varying the ambient temperature of B1 up to $10 \%$, the estimated concentration in the monitor point at $10 \mathrm{~m}$ from the release point at 0.2 high varies at most $0.7 \%$.

Table III - Concentration variation on B1

\begin{tabular}{c|cccc}
\hline \multirow{2}{*}{$\begin{array}{c}\text { Distance } \\
{[\mathrm{m}]}\end{array}$} & \multicolumn{2}{|c}{ Percentage changes by each parameter on B1 } \\
\cline { 2 - 5 } & $\begin{array}{c}\text { Tempera- } \\
\text { ture }\end{array}$ & $\begin{array}{c}\text { Wind } \\
\text { speed }\end{array}$ & $\begin{array}{c}\text { Ground } \\
\text { roughness }\end{array}$ & $\begin{array}{c}\text { Atmospheric } \\
\text { pressure }\end{array}$ \\
\hline 10 & $-0,7 \%$ & $-4,4 \%$ & $0,0 \%$ & $-2,2 \%$ \\
\hline 15 & $-0,7 \%$ & $-2,3 \%$ & $-0,3 \%$ & $-1,0 \%$ \\
\hline 20 & $-0,4 \%$ & $+1,8 \%$ & $-0,4 \%$ & $+0,7 \%$ \\
\hline 30 & $+0,4 \%$ & $-0,9 \%$ & $0,0 \%$ & $-0,9 \%$ \\
\hline 40 & $-0,5 \%$ & $-0,5 \%$ & $-0,5 \%$ & $-1,1 \%$ \\
\hline 50 & $-0,7 \%$ & $-0,7 \%$ & $-0,7 \%$ & $-2,0 \%$ \\
\hline 60 & $0,0 \%$ & $+0,8 \%$ & $0,0 \%$ & $-1,6 \%$ \\
\hline 70 & $0,0 \%$ & $+0,9 \%$ & $0,0 \%$ & $-1,8 \%$ \\
\hline 80 & $0,0 \%$ & $-1,0 \%$ & $-1,0 \%$ & $-3,1 \%$ \\
\hline 100 & $-1,3 \%$ & $-1,3 \%$ & $-1,3 \%$ & $-3,9 \%$ \\
\hline
\end{tabular}

Table IV - Variation in the estimated values on B2

\begin{tabular}{c|cccc} 
& \multicolumn{3}{c}{ Percentage changes by each parameter on B2 } \\
\hline $\begin{array}{c}\text { Distance } \\
{[\mathrm{m}]}\end{array}$ & Temperature & $\begin{array}{c}\text { Wind } \\
\text { speed }\end{array}$ & $\begin{array}{c}\text { Ground } \\
\text { roughness }\end{array}$ & $\begin{array}{c}\text { Atmospheric } \\
\text { pressure }\end{array}$ \\
\hline 10 & $+0,8 \%$ & $+6,3 \%$ & $0,0 \%$ & $+4,7 \%$ \\
\hline 15 & $+1,0 \%$ & $+3,7 \%$ & $+1,3 \%$ & $+3,0 \%$ \\
\hline 20 & $+0,7 \%$ & $+0,7 \%$ & $0,0 \%$ & $+1,4 \%$ \\
\hline 30 & $+0,7 \%$ & $+2,2 \%$ & $0,0 \%$ & $+0,7 \%$ \\
\hline 40 & $+0,8 \%$ & $+3,2 \%$ & $+0,8 \%$ & $+2,4 \%$ \\
\hline 50 & $-0,9 \%$ & $-3,6 \%$ & $0,0 \%$ & $+3,6 \%$ \\
\hline 60 & $-1,0 \%$ & $-5,0 \%$ & $-1,0 \%$ & $+5,0 \%$ \\
\hline 70 & $0,0 \%$ & $-5,5 \%$ & $0,0 \%$ & $+5,5 \%$ \\
\hline 80 & $+1,2 \%$ & $+6,1 \%$ & $+1,2 \%$ & $+7,3 \%$ \\
\hline 100 & $+1,5 \%$ & $+7,4 \%$ & $+1,5 \%$ & $+8,8 \%$ \\
\hline
\end{tabular}


The same verification of the previous tables was performed for all monitor points at different heights and the sensitivity degree of each parameter was defined according to the maximum percentage changes in the simulated values of concentration. The variables sensitivity was considered "low" if the maximum percentage changes were less or equal to $2 \%$, "medium" if between 2 and 5\% and "high" if greater than $5 \%$, this criteria was applied in order to determine which variables are most sensitive to changes.

Table $\mathrm{V}$ presents the maximum variation caused on concentration for each input and the variables sensitivity degrees for both scenarios. The changes up to $10 \%$ in the temperature cause minor effects in the results, since this variation is not enough to represent a change in the atmospheric stability. However, it is important to note that in different scenarios in which the evaporation process may take longer (such in a case of pool formation), the influence of variations in the ambient temperature may increase, since it will directly affect the vaporization rate.

In addition, from Table $\mathrm{V}$, it is possible to note that changes up to $10 \%$ in the ground roughness do not affect the results significantly. However, in both scenarios, the variations of the wind speed and pressure cause more significant changes in the simulated concentration values.

Table V - Sensitivity degree

\begin{tabular}{lrc}
\hline Parameter & $\begin{array}{c}\text { Maximum varia- } \\
\text { tion on results }\end{array}$ & $\begin{array}{c}\text { Sensitivity } \\
\text { degree }\end{array}$ \\
\hline Ambient temperature & $1.3 \%$ & Low \\
\hline \multirow{\infty}{*}{ Wind speed } & $4.4 \%$ & Medium \\
\cline { 2 - 3 } Ground roughness & $1.3 \%$ & Low \\
\hline Atmospheric pressure & $3.9 \%$ & Medium \\
\hline \multirow{\infty}{*}{ Ambient temperature } & $1.5 \%$ & Low \\
\hline Wind speed & $7.4 \%$ & High \\
\hline Ground roughness & $1.5 \%$ & Low \\
\hline Atmospheric pressure & $8.8 \%$ & High \\
\hline
\end{tabular}

Figure 3 shows the effect on concentration of varying wind speed in scenario B1. Simulated concentrations in each monitor point at height $0.2 \mathrm{~m}$ are plotted: the yellow bars represent the simulated values for the baseline scenario B1 using the initial values of Table I, the green bars represent the simulated values obtained using the wind speed value decreased by $10 \%$ and the blue bars represent the results obtained using the wind speed value increased by $10 \%$.

As can be observed, the effects of variations on wind speed are greater in near field and decrease on far field, being the effect anti-symmetric on near field and tending to a symmetric effect on far field; this occurs because near the source term there is more turbulence due to the jet and therefore there are more eddies generated by this turbulence. The wind contributes to the eddies formation and consequently to the cloud dilution. In far field, there is less turbulence; thus, the wind contributes less to cloud dilution and more to the cloud displacement, moving the entire cloud in the wind direction. This effect would probably be different in a scenario with pool formation; in such a case, there is less turbulence near the source and the wind contributes to cloud displacement and influences the evaporation rate of the pool.

Like Figure 3 for scenario B1, Figure 4 shows the simulated concentrations in each monitor point at height $0.2 \mathrm{~m}$ for the baseline scenario B2; it can be observed that the variations in the wind speed cause noticeable effects on the simulated values both on near and far field.

In scenario B2 the variations of wind speed affected the results near the source term, by the same reasons of scenario B1; however, unlike scenario $\mathrm{B} 1$, in the far field the results were also significantly affected. In this case, the wind contributes do the cloud dilution in the region near the source, after few meters the turbulence decrease; however, the fence at $15 \mathrm{~m}$ blocks partially the cloud and causes turbulence, consequently the results sensitive to variations in wind speed increases. The difference between the results for the baseline scenario and the scenarios with increase and decreased wind speed remains approximately constant from immediately after the fence until the last monitor point.

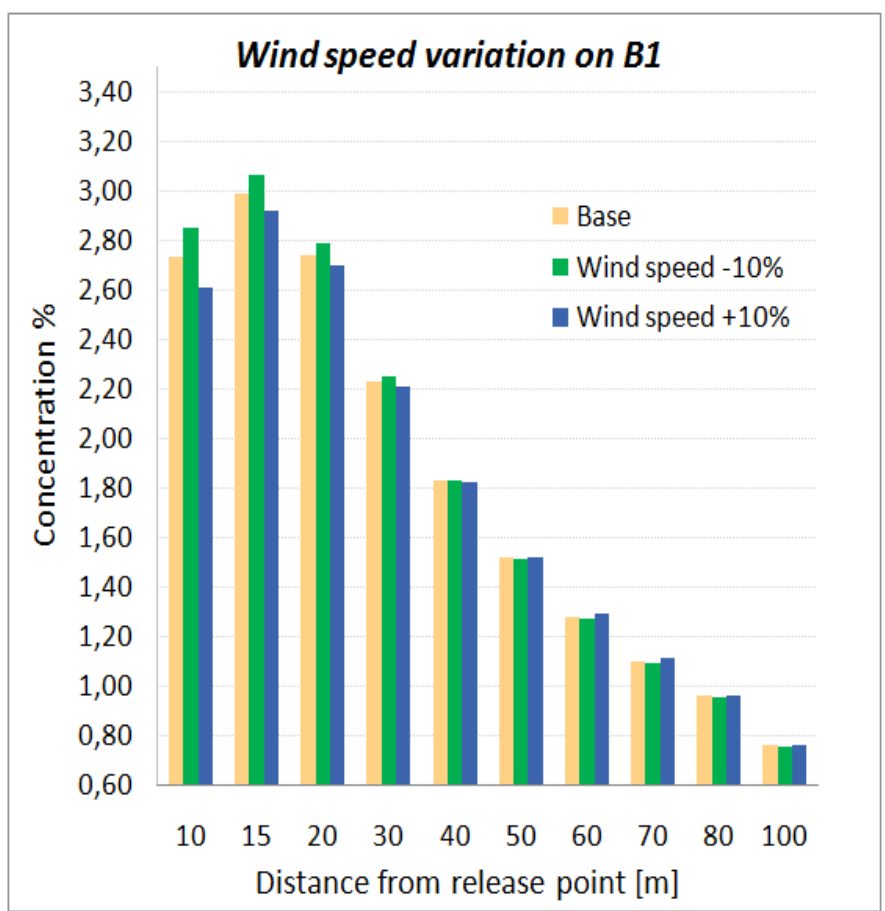

Figure 3 - Simulated concentrations varying wind speed on scenario B1 


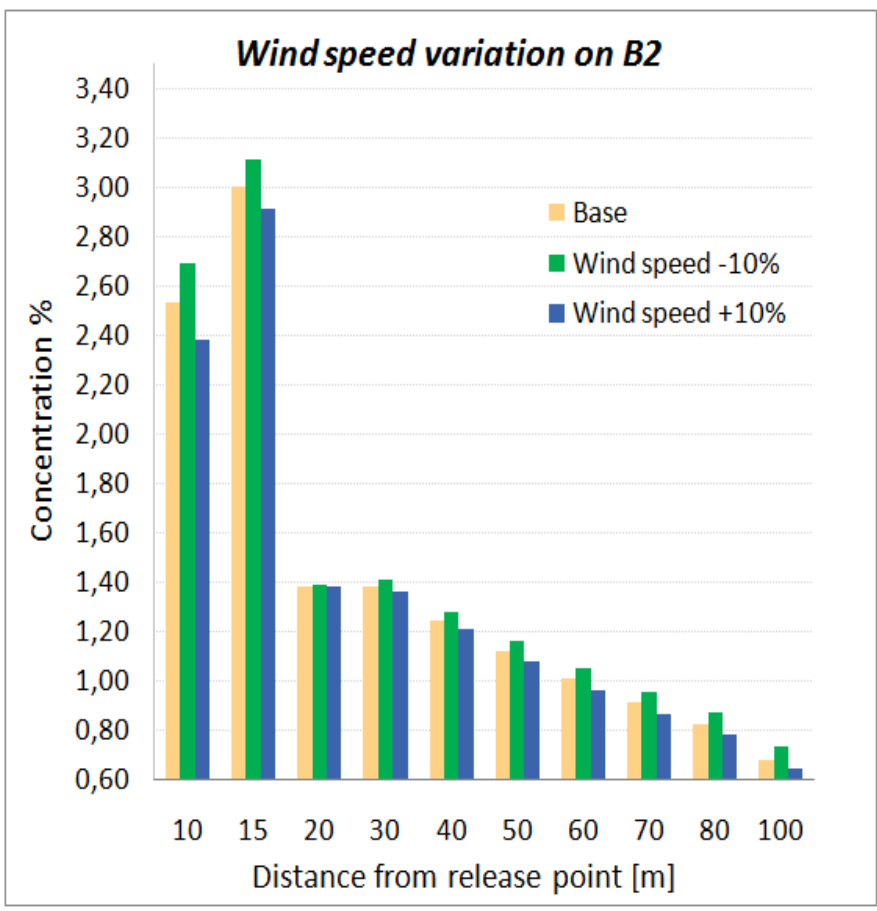

Figure 4 - Simulated concentrations varying wind speed on scenario B2

Concerning to variations in the pressure atmospheric values, Figure 5 and Figure 6 show the simulated concentrations in each monitor point at height $0.2 \mathrm{~m}$ for scenarios B1 and B2 respectively: the yellow bars represent the simulated values for the baseline scenario using the initial values of Table I, the green represents the predict values obtained using the atmospheric pressure value decreased by $10 \%$ and the blue represent the results obtained using the atmospheric pressure value increased by $10 \%$.

When the atmospheric pressure value is increased by $10 \%$ the simulated values for concentration for the gas phase decrease and when the atmospheric pressure value is decreased by $10 \%$ the simulated values for concentration increase; this probably occurs because with a higher pressure the liquid fraction into the cloud takes longer to evaporate; from the results of the monitor points at different heights, it is possible to note that the influence of the pressure variations is lowest in the highest part of the cloud, where the liquid fraction is smaller.

\section{CONCLUSIONS}

This study evaluated the sensitivity analysis of cloud dispersion using a CFD tool; Four variables of interest were considered: ambient temperature, wind speed, ground roughness and atmospheric pressure.

In order make the analysis, two trials of the field tests performed by HSL (Butler \& Royle, 2001) were chosen; the former presenting an unobstructed scenario and the second with a barrier blocking the spread of the cloud.

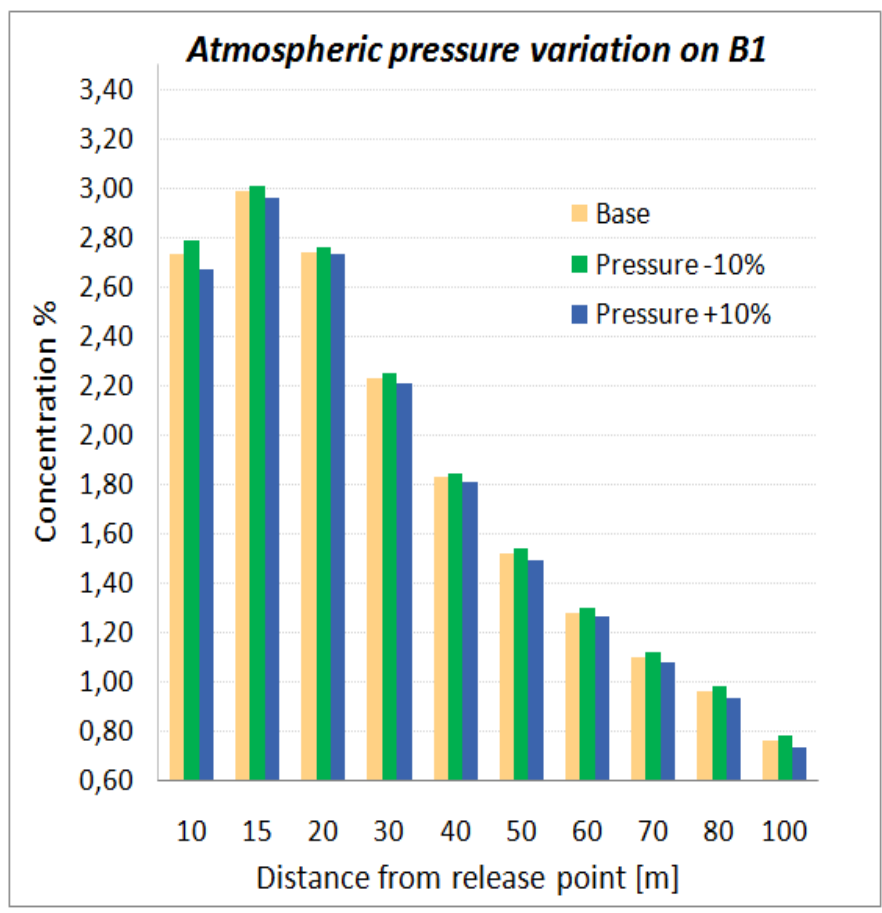

Figure 5 - Simulated concentrations varying atmospheric pressure on scenario B1

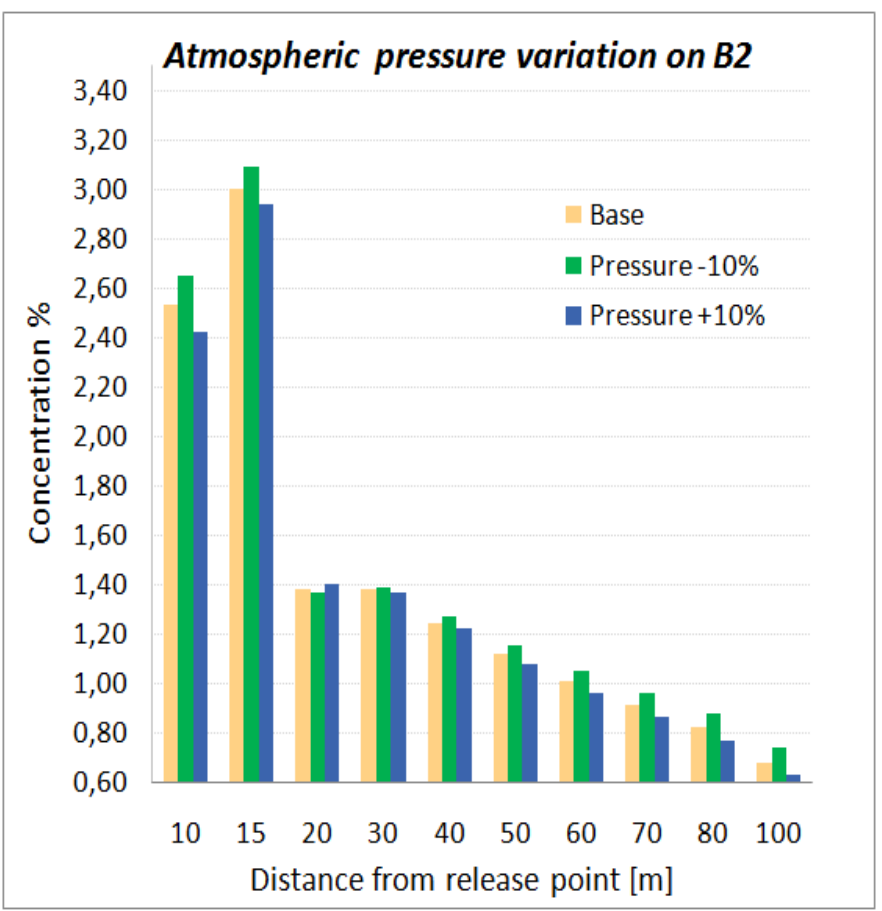

Figure 6 - Simulated concentrations varying atmospheric pressure on scenario $\mathrm{B} 2$

In both scenarios the results were more sensitive to changes in the values of the wind speed and atmospheric pressure. The variations on simulated concentrations reach up to $8.8 \%$; however, minor effects on results occurred due to changes in the ambient temperature and ground roughness, in these cases the variations on simulated results reach up to $1.5 \%$.

It is worth noting that the results showed a low sensitivity degree to temperature and roughness variations in the selected scenarios. However, this degree can be higher in other scenarios: for example, in scenarios in which there is pool formation, varia- 
tions in ambient temperature probably will influence more the results, since this parameter affects the evaporation process.

Moreover, the results were more sensitive to variations in wind speed and atmospheric pressure, showing that the accuracy of these values is essential to high quality dispersion modeling.

Future research will imply performing a sensitivity analysis of other key parameters on dispersion analysis, especially concerning source term features and other scenarios such as dispersion over water and pool formation. This will allow a better founded decision making process when setting computational parameters in CFD simulations.

\section{ACKNOWLEDGMENTS}

The authors gratefully wish to acknowledge the Program for Development of Human Resources (PRH) from Petrobras and Brazilian National Petroleum, Natural Gas and Biofuels Agency (ANP) by the financial support.

\section{REFERENCES}

Anderson, J. D. Jr. 1995. Computational Fluid Dynamics - The basics with applications. Maryland: McGraw-Hill. pp. 547.

CCPS - Center for Chemical Process Safety, 1995. Understanding Atmospheric Dispersion of Accidental Releases. New York: Center for Chemical Process Safety.

Coldrick, S., Lea, C. J., \& Ivings, M. J., 2009. Validation database for evaluating vapor dispersion model for safety analysis of LNG facilities - Review. The fire protection research foundation. Health and Safety Laboratory - HSE.

Cormier, B. R. 2008. Application of computational fluid dynamics for LNG vapor dispersion. Journal of Loss Prevention in the Process Industries. pp. 332-352.

Dharmavaram, S., Hanna, S. R., \& Hansen, O. R. 2005. Consequence Analysis-Using a CFD model for industrial Sites. Process Safety Progress, 24, pp. 316-327.

Gant, S. E., Kelsey, A., McNally, K., Witlox, H. 2013. Sensitivity Analysis of Dispersion Models for Jet Releases of Dense-Phase Carbon Dioxide. Chemical Engineering Transactions, 31, pp. 1-6.

Gavelli, F., Scott, D. G., \& Hansen, O. R. 2011. Evaluating the potencial for overpressures from the ignition of a LNG vapor cloud during offloading. Journal of Loss Prevention in the process industries, 24, pp. 908-915.

GexCon AS. 2014. FLACS Software - version 10.1.

HSE - Health and Safety Executive. 2010. Review of FLACS version 9.0 - Dispersion modelling capabilities. Available at: http://www.hse.gov.uk/research/rrpdf/rr779.pdf, accessed on January, 2013.

Launder, B. E., \& Spalding, D. B. 1974. The numerical computation of turbulent flows., 3, pp. 269-289.

Middha, P. and Hansen, O. R. 2009. Using computational fluid dynamics as a tool for hydrogen safety studies. Journal of Loss Prevention in the Process Industries. Vol. 22, pp. 295302.

Pandya, N., Gabas, N., Marsden, E. 2012. Sensitivity analysis of Phast's atmospheric dispersion model for three toxic materials (nitric oxide, ammonia, chlorine). Journal of Loss Prevention in the Process Industries(25), pp. 20-32.
Plasmans, J., Donnat, L., Carvalho, E., Debelle, T., Marechal, B., Baillou, F. 2012. Challenges with the use of CFD for major accident dispersion modeling. Process Safety Progress. Vol.00, pp. 1-5. doi: 10.1002/prs.11571.

Schleder, A. M., Martins, M. R. 2013. The use of Integrals and CFD tools to evaluate the cloud dispersion of flammable and toxic substances leakages. In: European Safety and Reliability Congress - ESREL, 2013, Amsterdã. Proceedings of ESREL 2013 - European Safety and Reliability Congress. pp. 1-8.

Schleder, A. M., Martins, M. R., Pastor, E., Planas, E. 2014. The effect of the computational grid size on the prediction of a flammable cloud dispersion. In: International Conference on Ocean, Offshore and Arctic Engineering, 2013, San Francisco, California, USA. Proceedings of the ASME 2014 33th International Conference on Ocean, Offshore and Arctic Engineering, 2014. pp. 1-8.

TNO - The Netherlands Organization of Applied Scientific Research 2005. Methods for the calculation of physical effects due to releases of hazardous materials (liquids and gases) Yellow Book (Third Edition ed.). Committee for the prevention of disasters. 